\title{
Effects of Hydrocortisone Administration on Psychological State and Beliefs: Pre-Analysis Plan
}

\author{
Prachi Jain and Johannes Haushofer
}

January 9, 2017

\begin{abstract}
This document describes the analysis plan for a randomized, double-blind laboratory experiment examining the effects of hydrocortisone administration on a variety of behavioral outcomes. Our study includes 120 subjects from the informal settlements in Nairobi, Kenya. Using a double-blind procedure, respondents are randomly administered either a placebo or a $20 \mathrm{mg}$ dose of hydrocortisone, a drug that increases the levels of the stress hormone cortisol, for seven consecutive days. This plan outlines the design of the study, the outcomes of interest, and the econometric approach.
\end{abstract}




\section{Introduction}

The present project studies the effect of hydrocortisone administration on measures of psychological state and beliefs about drug condition. Specifically, we test whether $20 \mathrm{mg}$ of hydrocortisone have effects relative to placebo on positive and negative affect, state anxiety, perceived stress, and depression symptoms. In addition, we ask whether participants are able to guess the identity of the drug they received. To test whether "acute" and "chronic" stress have different effects on these outcomes, we administer hydrocortisone or placebo for 7 consecutive days, and compare behavioral responses on the first vs. the seventh day. The stress literature has long distinguished between these two types of stress (McEwen, 2004), and it has recently been shown that risk aversion is affected by chronic but not acute stress (Kandasamy et al., 2014); however, little is known about the relative effects on other outcomes. We hasten to add that we cannot hope to mimic the effects of truly chronic stress (over years) with this manipulation; however, it has been shown that the effects of stress differ, both behaviorally and neurobiologically, even over short periods such as several hours (Henckens et al., 2010, 2011; Joëls et al., 2011) or weeks (Kandasamy et al., 2014; Rubio et al., 1989).

\section{Design}

\subsection{Sampling Strategy}

We study a sample of Nairobi residents registered as participants with the Busara Center for Behavioral Economics. To be registered in the Busara participant pool, respondents must be over the ages of 18 years old, have access to a mobile phone, and have access to MPesa, a mobile money system used for payment of respondents.

The study took place in September and October 2016 with a sample of 120 participants, with about half in each the treatment and control group. Prior to the full study, we completed pilots with a sample of 32 respondents to finalize the relatively complicated protocols, and identify potential difficulties in the main experiment. Due 
to the resulting changes in protocol for the main study, the data from these 32 pilot participants will not be included in the analysis described below.

Participants were screened for inclusion/exclusion criteria both over the phone and in person. We restricted our sample to respondents in the Busara participant pool between the ages of 18 and 40 who had at least 8 years of education to ensure literacy. We excluded participants who did not meet the requirements of an extensive health screening. 1

To mitigate factors that might affect measurement of salivary cortisol, we asked that participants not drink alcohol, drink coffee, or smoke on the days of the study and the day before the study began. Also, we asked that participants not eat, drink liquids other than water, or engage in strenuous physical activity, including sexual activity, during the 2 hours before the study.

\subsection{Treatment}

Participants in the treatment group received $20 \mathrm{mg}$ of oral hydrocortisone; participants in the placebo group received a placebo that was identical in appearance.

\subsection{Data Collection}

We conducted laboratory session with approximately 20 respondents per session, until we reached 120 respondents. Before each session began, we generated participant IDs, each of which was randomly assigned to the treatment or placebo group. At the beginning of each session, each participant was screened by a nurse and was randomly assigned a participant ID. The study was double-blind, such that both the laboratory staff and participants were unaware of their treatment status. The remainder of the sessions followed the schedule of tasks and treatments outlined below.

The treatments, tasks, and questionnaires are administered using touch screen computers to enable computer-illiterate respondents to participate. Enumerators read instructions to the respondents in English and Swahili to maximize comprehension. Respondents received a cash compensation of 350 KES on each day, plus

\footnotetext{
${ }^{1}$ Our exclusion criteria can be provided upon request.
} 
a $50 \mathrm{KES}$ for arriving on time. In addition, participants received $400 \mathrm{KES}$ on the first day of participation, another $300 \mathrm{KES}$ on the seventh day of participation, and another $500 \mathrm{KES}$ if they attend all seven days of the study. In addition, participants could receive an additional $100 \mathrm{KES}$ for correctly guessing their treatment status. The compensation and bonus were transferred to the respondents via MPesa.

\subsection{Schedule of Tasks and Treatments}

Days 1 and 7 (psychological and other self-reported outcomes are in italics):

1. Welcome

2. Consent and Nurse's Checklist

3. Nurse's Meeting for Participant Inclusion

4. Heart Rate and Blood Pressure Measurement

5. Introduction to Computer Interface

6. PANAS

7. Salivette 1: baseline

8. Self-Reported Stress Question 1

9. Placebo/Hydrocortisone Administration

10. Anxiety STAIT Questionnaire

11. Salivette 2

12. Self-Reported Stress Question 2

13. Heart Rate and Blood Pressure Measurement

14. Salivette 3 
15. Self-Reported Stress Question 3

16. Anxiety STAIS

17. PANAS

18. Salivette 4

19. Self-Reported Stress Question 4

20. Perceived Stress Scale

21. Salivette 5

22. Self-Reported Stress Question 5

23. Depression CESD

24. Cantril

25. Salivette 6

26. Self-Reported Stress Question 6

27. General Questionnaire

28. Salivette 7

29. Self-Reported Stress Question 7

30. Heart Rate and Blood Pressure Measurement

31. Guessing Module

32. Debrief 


\section{Days 2-6}

1. Welcome

2. Consent and Nurse's Checklist

3. Questionnaire (Day 2: Discounting, Day 3: Executive Control, Day 4: SelfEfficacy)

4. Heart Rate and Blood Pressure Measurement

5. Placebo/Hydrocortisone Administration

6. Heart Rate and Blood Pressure Measurement

7. PANAS

8. General Questionnaire

9. Guessing Module

10. Debrief

\section{Econometric Approach}

\subsection{Main Treatment Effects}

We begin by asking whether hydrocortisone affects our outcomes of interest, separately for the acute and chronic treatments. To this end, we restrict the subsample to either day 1 or day 7, and test whether participants administered hydrocortisone respond differently on a variety of outcomes (to be described below) than participants given placebo pills, using the following specification:

$$
y_{i}=\beta_{0}+\beta_{1} T_{i}+\varepsilon_{i}
$$


Here, $y_{i}$ is the outcome of interest for respondent $i$. Note that for some outcome variables, $y_{i}$ is measured in first differences (see below). $T_{i}$ is a treatment indicator that takes value 1 for respondents that received the hydrocortisone pill and 0 for respondents that receive the placebo pill. Thus, $\beta_{1}$ estimates the treatment effect of hydrocortisone on each outcome.

In addition, we want to examine the effects of chronic stress on behaviors, and whether chronic stress differentially affects behaviors relative to acute stress. To examine the former, we will use the specification above and instead restrict our subsample to day 7 . To examine the latter, we will use the following empirical specification with a sample of outcomes from both days 1 and 7 .

$$
y_{i}=\beta_{0}+\beta_{1} T_{i}+\beta_{2} \operatorname{Day} 7+\beta_{3} T_{i} \cdot \operatorname{Day} 7+\varepsilon_{i t}
$$

where $y_{i t}$ is the outcome of interest for respondent $i$ on Day $7 . T_{i}$ is a treatment indicator that takes value 1 for respondents that receive the hydrocortisone pill and 0 for respondents that receive the placebo pill (recall that treatment is constant across the week). Day7 indicates whether the observation is from day 7 of the study (as opposed to Day 1). Thus, $\beta_{1}$ estimates the treatment effect of hydrocortisone on each

outcome, $\beta_{2}$ indicates whether there is day fixed effect on outcomes, and $\beta_{3}$ estimates whether hydrocortisone has a different effect on day 7 (chronic) than on day 1 (acute). Therefore, $\beta_{3}$ is our primary coefficient of interest in this specification. Note that if participants differentially show up to Day 7 sessions by treatment, the coefficient $\beta_{3}$ may be biased. For example, if the participants who receive hydrocortisone for 7 days and have a higher response to the stressor are less likely to show-up on Day $7, \beta_{3}$ would be biased downwards. We therefore restrict the sample to participants who showed up for at least 5 days of the study and were present on day 7 .

\subsection{Outcomes of Interest}

\section{Balance, integrity, and manipulation check}

(a) Randomization Check: To determine whether randomization was suc- 
cessful, we estimate our main equations with a number of demographics as outcome variables, including age, gender, years of education, average earnings and earnings after spending per month, BMI, number of siblings and dependents, whether the respondent depended on someone else financially, current unemployment, whether currently in debt, and perceived standing of the respondent in their community.

(b) Selection into Chronic Treatment: To test for differential attrition by treatment, we regress whether the participant was present on day 7 on treatment assignment, using equations 1 and 2.

Second, equation 3 assesses whether attriting individuals are different in terms of the demographics described above.

$$
y_{i}=\beta_{0}+\beta_{1} a_{t r i t}+\varepsilon_{i}
$$

We will also estimate this equation using baseline levels of cortisol and self-reported stress, and their responses to hydrocortisone treatment on day 1 , as outcome variables.

Third, equation 4 measures whether the baseline characteristics of attriting individuals in the treatment group are significantly different from those in the placebo group. The sample for this regression will be restricted to attrition households:

$$
y_{i}=\beta_{0}+\beta_{1} T_{i}+\varepsilon_{i}
$$

(c) Cortisol: We measure salivary cortisol at seven points during the sessions on day 1 and during those on day 7 . The first salivette is taken before administration of the pills. The second through seventh salivettes samples are taken at 25 minute intervals after the pills are administered. Cortisol samples are analyzed by Lancet Labs in Nairobi. We compute the area under the curve before analysis, such that the seven measurements provided by each participant in each session are collapsed to one number. Because cortisol levels are noisy, we include specifications in which we 
top-code the cortisol variable at the 99th percentile. In addition, cortisol levels are affected by time of day, smoking, consumption of food and drink, and exercise. We attempt to mitigate the influence of these factors by asking participants not to smoke, not to drink alcohol, tea, or coffee; and we ask participants not to eat or drink, nor to performance intense exercise within 2 hours of the sessions. However, participants may not always follow these rules; we therefore survey them on whether they did these activities, and include specifications control variables for these activities on the right-hand side. Thus, we will analyze six versions of the cortisol variable: with and without top-coding, crossed with inclusion or omission of control variables.

Time Path of Cortisol: To examine the time path of cortisol in more detail, we restrict the sample to either Day 1 or Day 7 and use the following empirical specification:

$$
y_{i}=\beta_{0}+\sum_{s \in\{1 \ldots 7\}} \gamma_{s} \cdot \mathbb{1}(\text { Sample }=s)+\sum_{s \in\{1 \ldots 7\}} \beta_{s} T_{i} \cdot \mathbb{1}(\text { Sample }=s)+\varepsilon_{i}
$$

where $y_{i}$ is the measure of salivary cortisol for respondent $i$ in saliva sample $s . T_{i}$ is a treatment indicator that takes value 1 for respondents that receive the hydrocortisone pill and 0 for respondents that receive the placebo pill (recall that treatment is constant across the week). Sample indicates the number of the saliva sample (1-7).

\section{Main outcomes}

In the following, we describe the main outcome variables of interest. Outcome variables we consider primary and which will enter the correction for multiple comparisons are marked with asterisks.

(a) Self-Reported Stress*: Throughout the experiment, we ask participants to indicate (on a scale of 0-100) "In the present moment, I feel stressed." The question is asked after each salivette. As described under 
(a), we will compute the area under the curve for this outcome measure. In addition, we will estimate the detailed specification given in equation 5 . We will not include control variables or top-code because this self-report variable does not have the same outlier concerns and confounds as cortisol.

(b) PANAS*: To measure positive and negative general affect states, we generated a Kiswahili version of the PANAS (Positive and Negative Affect Schedule). On a scale of 0-100, respondents indicated how they felt in the present moment for each of 20 emotions. We asked this series of the questions at least once on each day (days 1 through 7 ) of the experiment. On days 1 and 7, respondents completed the PANAS twice: before administration of the pill, and approximately 60 minutes after administration of the pill. These variables will be analyzed in first differences. We will analyze each of the 20 items individually, and create overall scores for positive and negative affect. Although we collected data on the PANAS on days 2 through 6 , we will not include this data for our analysis since the translation of the PANAS differed from days 1 and 7 due to a translation error.

(c) STAI-S*: The State Anxiety Inventory includes 20 items for assessing state anxiety in which the respondent indicates, on a scale from 0 to 100 , how they feel in the present moment (for example, "In the present moment I feel upset.") We ask the series of questions approximately 50 minutes after administration of the pill on Days 1 and 7 . The variables will be analyzed in levels, both as individual questions and as an overall score.

(d) Cohen's Subjective Stress Scale: The CPSS was developed to measure the degree to which situations in one's life are perceived as stressful. The respondent indicates, on a scale from 0 to 100, how often in the past week they have felt a certain way (for example, "In the past week, how often have you felt unable to control the important things in your life."). We ask the series of questions approximately 100 minutes after administration of the pills. The variables will be analyzed in levels, both as 
individual questions and as an overall score. This variable does not enter the correction for multiple comparisons because it asks participants about the past week, rather than their current psychological state.

(e) CESD: The Center for Epidemiological Studies Depression scale includes 20 items for assessing how often over the past week a respondent indicates experiencing symptoms associated with depression. The respondent indicates, on a scale of 0 to 100 , how often in the past week they have felt a certain way (for example, "In the past week I did not feel like eating; my appetite was poor.") We ask the series of questions approximately 125 minutes after administration of the pill. The variables will be analyzed in levels, both as individual questions and as an overall score. This variable does not enter the correction for multiple comparisons because it asks participants about the past week, rather than their current psychological state.

(f) Cantril's Self-Anchoring Scale: The respondent indicates, on a scale of 0 to 10 , where they currently perceive themselves on a ladder, where the top of the ladder represents the best possible life for the respondent. In addition, the respondent answers where they imagine themselves standing in five years. The variables will be analyzed in levels as individual questions. This variable does not enter the correction for multiple comparisons because it asks participants about an extended current state, rather than their momentary state.

(g) Guessing drug condition: In a guessing module, participants were asked which pill they think they received, and indicated how confident they were that they received that pill. The outcome of interest is whether the respondent guessed correctly $\bigsqcup^{2}$

To assess whether participants could correctly guess which pill they received, we construct a variable for correctly guessing the identity of the

\footnotetext{
${ }^{2}$ In addition, participants are asked what their guesses about the identity of the pill they received was based on: the taste of the pill, look of the pill, physiological reactions, psychological reactions, belief that more participants received one type of pill over the other, or picked at random.
} 
pill, regress it on the constant, and test whether the coefficient differs from chance performance.

\section{Other outcomes}

(a) Heart Rate and Blood Pressure: Heart rate and blood pressure are measured at 3 points during the experiment: before the pill, following the pill, and approximately 150 minutes after ingestion of the pill. These measures are taken for safety reasons and are not manipulation checks because they are not timed to capture peak cortisol levels. We analyze them for the sake of completeness, using as dependent variables the pairwise differences between the three measurement timepoints.

\subsection{Dimensions of heterogeneity}

We will estimate heterogeneous treatment effects using versions of our main estimating equations in which we add a main effect and interaction terms for a binary interactant of interest. We consider the following dimensions of heterogeneity:

1. Gender

2. Above median baseline depression level

3. Above median baseline stress level

4. Above median baseline cortisol level

5. Above median baseline Trait Anxiety

\subsection{Accounting for multiple inference}

We will correct across the outcomes marked with asterisks above using the false discovery rate (FDR) following Anderson (2008). 


\section{References}

[1] Anderson, Michael L. 2008. "Multiple Inference and Gender Differences in the Effects of Early Intervention: A Reevaluation of the Abecedarian, Perry Preschool, and Early Training Projects." Journal of the American Statistical Association, 103 (484): 1481-1495.

[2] Henckens, Marloes J.A.G., Guido A. van Wingen, Marian Joëls, and Guillén Fernández. 2010. "Time-dependent effects of corticosteroids on human amygdala processing. " The Journal of Neuroscience, 30 (38): 12727-12732.

[3] Henckens, Marloes J.A.G., Guido A. van Wingen, Marian Joëls, and Guillén Fernández. 2011. "Time-dependent corticosteroid modulation of prefrontal working memory processing. "Proceedings of the National Academy of Sciences, 108 (14): 5801-5806.

[4] Joëls, Marian, Guillén Fernández and Benno Roozendaal. 2011. "Stress and emotional memory: a matter of timing. "Trends in Cognitive Sciences, 15 (6): $280-288$.

[5] Kandasamy, Narayanan, Ben Hardy, Lionel Page, et al. 2014. "Cortisol shifts financial risk preferences." Proceedings of the National Academy of Sciences, 111 (9): 3608-3613.

[6] McEwen, Bruce S. 2004. "Protection and Damage from Acute and Chronic Stress: Allostasis and Allostatic Overload and Relevance to the Pathophysiology of Psychiatric Disorders." Annals of the New York Academy of Sciences, 1032: $1-7$.

[7] Rubio, C.A. M. Sveander, A. Duvander. 1989. "A model to evaluate acute and chronic stress in the colonic mucosa of rats." Annals of the New York Academy of Sciences, 32 (1): 26-29. 